\title{
Tromelin Island
}

National Cancer Institute

\section{Source}

National Cancer Institute. Tromelin Island. NCI Thesaurus. Code C123771.

An island in the Indian Ocean east of Madagascar. 